\title{
Inhibition of the Hedgehog pathway induces autophagy in pancreatic ductal adenocarcinoma cells
}

\author{
YONGHUA XU ${ }^{1 *}$, YONG AN $^{2 *}$, XUEHAO WANG ${ }^{3}$, WENZHANG ZHA ${ }^{1}$ and XIANGCHENG $\mathrm{LI}^{3}$ \\ ${ }^{1}$ Department of General Surgery, Fourth Affiliated Hospital of Nantong University, The First People's Hospital of Yancheng, \\ Yancheng, Jiangsu; ${ }^{2}$ Hepatobiliary Surgery, The First People's Hospital of Changzhou, Changzhou, Jiangsu; \\ ${ }^{3}$ Liver Transplantation Center, First Affiliated Hospital of Nanjing Medical University, Nanjing, Jiangsu, P.R. China
}

Received September 16, 2013; Accepted November 4, 2013

DOI: $10.3892 /$ or.2013.2881

\begin{abstract}
The HH signaling pathway is a 'core' signal transduction pathway in pancreatic cancer that promotes the tumorigenesis of pancreatic cancers via enhancing cell proliferation, increasing invasion and metastasis and protecting against apoptosis. In the present study, we found that $\mathrm{HH}$ signaling regulates autophagy in pancreatic cancer cells. Activation of $\mathrm{HH}$ signaling inhibits autophagy, while inhibition of the $\mathrm{HH}$ pathway induces autophagy. Although the role of autophagy in cell survival and apoptosis may depend on tumor type and the microenvironment, our data clearly demonstrated that GANT61-induced autophagy contributed to reduced viability and increased apoptosis in pancreatic cancer cells both in vivo and in vitro, and these effects were reversed by the autophagy inhibitor, 3-MA. We propose that $\mathrm{HH}$ signaling by regulating autophagy plays an important role in determining the cellular response to $\mathrm{HH}$-targeted therapy in pancreatic cancer and further investigation of the interaction between autophagy and $\mathrm{HH}$ signaling is particularly important.
\end{abstract}

\section{Introduction}

Pancreatic ductal adenocarcinoma (PDAC) is one of the most lethal of all human malignancies (1). Great improvements have been made in surgical and chemotherapeutic approaches during the past decades; however, the survival rate of patients

Correspondence to: Professor Wenzhang Zha, Department of General Surgery, Fourth Affiliated Hospital of Nantong University, The First People's Hospital of Yancheng, Yancheng, Jiangsu, P.R. China E-mail: zhawenzhang@sohu.com

Professor Xiangcheng Li, Liver Transplantation Center, First Affiliated Hospital of Nanjing, Medical University, 300 Guanzhou Road, Nanjing, Jiangsu, P.R. China

E-mail:drxcli@njmu.edu.cn

*Contributed equally

Key words: Hedgehog signaling, autophagy, GANT61, pancreatic cancer, apoptosis with PDAC has not been significantly prolonged. Therefore, an alternative therapy for PDAC is urgently required.

The Hedgehog $(\mathrm{HH})$ signaling pathway is an essential pathway during embryonic development (2). Sonic Hedgehog (Shh) is a secreted mammalian ortholog of the Hedgehog family and plays multiple roles during embryonic development (3). Activation of the Shh pathway triggers a series of intracellular events through the GLI transcriptional effectors Gli-1, Gli-2 and Gli-3 (4,5).

The HH pathway is reactivated in tumor development, and plays an important role in the tumorigenesis and maintenance of tumors (6-8), including pancreatic cancer (9). Evidence has shown that the $\mathrm{HH}$ signaling pathway is a 'core' signal transduction pathway in pancreatic cancer and is abnormally expressed in almost all pancreatic cancer cells (10). Accumulating data suggest that the $\mathrm{HH}$ pathway promotes the tumorigenesis of pancreatic cancers by enhancing cell proliferation $(11,12)$, increasing invasion and metastasis $(13,14)$ and protecting against apoptosis $(15,16)$.

In a phase I trial testing vismodegib (GDC-0449), an inhibitor of Smoothened (SMO), beneficial responses were observed in patients with advanced basal cell carcinoma (BCC) and medulloblastoma. However, only one in eight patients with pancreatic carcinoma experienced a stable disease as the best response (17). The ineffectiveness of a pathway inhibitor for pancreatic cancer can probably be explained by the fact that there are numerous crosstalks between the $\mathrm{HH}$ signaling pathway and other signaling pathways in pancreatic cancer (18-20). Feedback from these crosstalk pathways may reduce the therapeutic effectiveness of the $\mathrm{HH}$ pathway inhibitor. Therefore, further investigation should be focused on the mechanism of interaction between $\mathrm{HH}$ signaling and these crosstalk pathways.

Autophagy was initially reported 50 years ago by Ashford and Porter (21), and it has recently gained considerable attention. Autophagy is a lysosome-mediated protein and organelle degradation process that is characterized by the formation of double-membrane vesicles, referred to as autophagosomes $(22,23)$. Autophagy is involved in several pathophysiological processes and contributes to numerous diseases, particularly to cancer $(24,25)$. However, the function of autophagy in cancer has yet to be fully clarified, as it acts both as a tumor suppressor and as a tumor promoter (26). 
During the development of PDAC, autophagy initially suppresses tumor initiation yet supports tumor growth at later stages $(27,28)$. Other studies have reported that pancreatic cancers require autophagy for tumor growth (29) and that autophagy is correlated with poor outcome in PDAC patient (30). Many results suggest that autophagy is a cell death mechanism in response to drugs such as gemcitabine and triptolide. It was confirmed that autophagy is activated by gemcitabine and triptolide in the treatment of pancreatic cancer cells and that activated autophagy plays a role in cancer suppression $(31,32)$. However, it was also reported that activation of autophagy by receptor for advanced glycation end products (RAGE) $(33,34)$, 2-deoxy-D-glucose (2-DG) (35), phosphatidylinositol 3-kinase (PI3K) (36) and Kangai 1 (KAI1) (37) promotes tumorigenesis and limits apoptosis in pancreatic cancer cells. Hence, the role and mechanism of autophagy is unclear in pancreatic cancer and requires further investigation.

Although HH signaling is known to inhibit apoptosis, it remains unknown whether $\mathrm{HH}$ signaling is able to regulate autophagy in pancreatic cancer cells. The present study describes a novel role of the HH signaling pathway in the regulation of autophagy in PDAC cells. We report that inhibition of the $\mathrm{HH}$ pathway induces autophagy and enhances apoptosis in PDAC cells. Our findings suggest that the status of autophagy is a key factor that may influence the cellular response to $\mathrm{HH}$ signaling-targeted therapy.

\section{Materials and methods}

Cell cultures. Human pancreatic cancer cell line CFPAC-1 was purchased from the Shanghai Cell Bank (Shanghai, China) and propagated in our laboratory by culturing in Dulbecco's modified Eagle's medium (DMEM) (Invitrogen, Carlsbad, CA, USA) with $10 \%$ fetal bovine serum (FBS) (Sigma, St. Louis, $\mathrm{MO}, \mathrm{USA}$ ) at $37^{\circ} \mathrm{C}$ with $5 \% \mathrm{CO}_{2}$, supplemented with $1 \%$ penicillin/streptomycin. BALB/c nude mice (4-week-old, female) were purchased from the Model Animal Research Center of Nanjing University, Nanjing, China. Drug treatment included recombinant human N-Shh (R\&D Systems, Minneapolis, MN, USA) used at $0.25 / 0.5 \mu \mathrm{g} / \mathrm{ml}$; GANT61 (Abcam, Cambridge, MA, USA) used at $5 / 10 \mu \mathrm{M}$ soluble in DMSO (dimethyl sulphoxide); and 3-MA (3-methyladenine) (Sigma) used at $5 \mathrm{mmol} / \mathrm{l}$.

Reverse transcription quantitative real-time polymerase chain reaction (RT- $q P C R)$ for $m R N A$ quantitation. Total RNA of cells was isolated using TRIzol reagent (Invitrogen). cDNA was synthesized using the PrimeScript RT reagent kit (Takara, Dalian, China). Quantitative RT-PCR was performed using an ABI 7500 (Applied Biosystems) with FastStart Universal SYBR-Green Master (Rox) (Roche) for mRNA quantitation and with the TaqMan ${ }^{\circledR}$ MicroRNA assay kit (Applied Biosystems). The relative expression of mRNA was calculated as the inverse log of the $\Delta \Delta \mathrm{Ct}$ (38) and normalized to $\beta$-actin. Primers for mRNA qPCR were synthesized by Invitrogen (Shanghai, China). The sequences were: Gli-1 sense, 5'-AGCC AAGCACCAGAATCGGAC-3' and antisense, 5'-GTTTGGTC ACATGGGCGTCAG-3'; Gli-2 sense, 5'-GGGTCTGGGGTC AGCCTTTGGA-3' and antisense, 5'-AATGGCGACAGGGT
TGACGGT-3'; $\beta$-actin sense, 5'-AGAAAATCTGGCACCAC ACC-3' and antisense, 5'-TAGCACAGCCTGGATAGCAA-3'.

Cell viability assays. Cell viability was determined using an MTT assay. Aliquots of $2 \times 10^{3}$ cells were planted in 96-well plates with $200 \mu \mathrm{l}$ culture medium per well accordingly, and the viability was measured at $72 \mathrm{~h}$ thereafter using the following procedures. Cells were incubated with $20 \mu \mathrm{l} /$ well of MTT solution ( $5 \mathrm{mg} / \mathrm{ml}$; Sigma) at $37^{\circ} \mathrm{C}$ for $4 \mathrm{~h}$. Then $150 \mu \mathrm{l}$ of DMSO was substituted for the supernatant, followed by oscillation for $10 \mathrm{~min}$. Absorbance at $490 \mathrm{~nm}$ was detected by the Multiskan MK3 microplate reader (Thermo Labsystems, Philadelphia, PA, USA). All results were normalized to corresponding controls and are expressed in terms of percentage.

Analysis of cell apoptosis. Cell apoptosis was assessed by flow cytometry (Becton-Dickinson, San Jose, CA, USA). For cell apoptosis, cells were treated with GANT61 $(10 \mu \mathrm{M}), 3$-MA $(5 \mathrm{mmol} / \mathrm{l})$ and a combination of GANT61 and 3-MA for $48 \mathrm{~h}$. Cells were then collected, washed, suspended in $100 \mu \mathrm{l} 1 \mathrm{X}$ binding buffer and stained with $5 \mu \mathrm{l}$ fluorescein isothiocyanate (FITC)-Annexin V and $1 \mu \mathrm{l} \mathrm{PI}$ at room temperature for $15 \mathrm{~min}$ in the dark. The stained cells were immediately analyzed by flow cytometry.

Western blotting. For western blotting, cells were treated with $400 \mathrm{nM}$ bafilomycin for $2 \mathrm{~h}$ before being lysed using RIPA buffer with $1 \%$ PMSF on ice. The concentration of total protein was determined using a BCA kit (Nanjing KeyGen Biotech., Co., Ltd., Nanjing, China). Equal amounts of protein $(30 \mu \mathrm{g})$ were resolved with $10 \%$ SDS-PAGE and transferred to polyvinylidene difluoride (PVDF) membranes (Millipore, Bedford, MA, USA) using a Mini Trans-Blot apparatus (BioRad Laboratories, Hercules, CA, USA). Membranes were probed with primary antibodies for $12 \mathrm{~h}$ at $4^{\circ} \mathrm{C}$ and then incubated with secondary antibodies for $2 \mathrm{~h}$ at room temperature. Primary antibodies used were LC3 (microtubule-associated protein light chain 3) (Novus Biologicals, Littleton, CO, USA) and tubulin (Santa Cruz Biotechnology, Santa Cruz, CA, USA) which was used as an internal control. The secondary antibody was purchased from Beyotime (Santa Cruz Biotechnology). Electrochemiluminescence was performed with a ChemiImager 5500 imaging system (Alpha Innotech Co., San Leandro, CA, USA).

Transmission electron microscopy. For ultrastructural examination, samples $\left(\sim 1 \mathrm{~mm}^{3}\right)$ were fixed with $2 \% \mathrm{OsO}_{4}$ and embedded in Araldite. Ultrathin sections were stained with uranyl acetate and lead citrate and inspected using an electron microscope (JEOL JEM-1010; Jeol, Tokyo, Japan).

Orthotopic xenograft tumor study. CFPAC- 1 cells $\left(2 \times 10^{6}\right)$ suspended in $100 \mu 1$ PBS were injected undercapsule of the pancreas in 4-week-old BALB/c nude mice. After 1 week, mice were randomized to three groups; vehicle, GANT61 $(50 \mathrm{mg} / \mathrm{kg})$, GANT61 $(50 \mathrm{mg} / \mathrm{kg})$ in combination with 3-MA $(10 \mathrm{mg} / \mathrm{kg})$ were administered every other day, i.p. for 28 days. At the end of the drug treatment, mice were euthanized and an autopsy was performed to obtain the primary lesions. Tumor volume was determined as $\mathrm{V}=\left(\mathrm{L} \mathrm{x} \mathrm{W}^{2}\right) / 2$, where $\mathrm{L}$ represents 

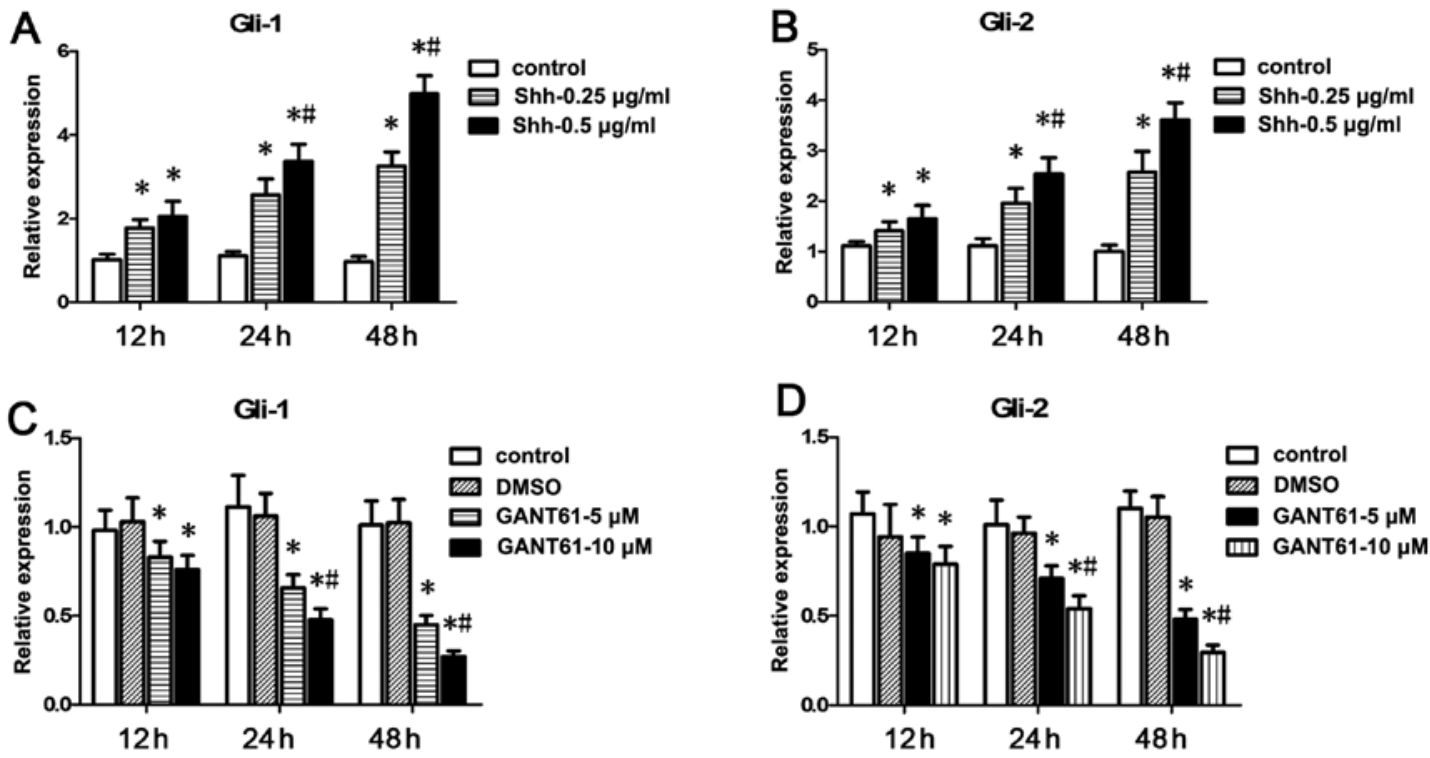

Figure 1. HH signaling is active in human pancreatic cancer CFPAC-1 cells. CFPAC-1 cells were treated with N-Shh $(0.25 / 0.5 \mu \mathrm{g} / \mathrm{ml})$ or GANT61 (5/10 $\mu \mathrm{M})$ for 12,24 and $48 \mathrm{~h}$, respectively. mRNA levels of Gli-1 and Gli-2 were confirmed by qRT-PCR. The relative levels were normalized to $\beta$-actin ("P<0.05 compared with the control group, $\mathrm{n}=3 ;{ }^{\#} \mathrm{P}<0.05$ compared with Shh- $0.25 \mu \mathrm{g} / \mathrm{ml}$ and GANT61-5 $\mu \mathrm{M}$ for Shh- $0.5 \mu \mathrm{g} / \mathrm{ml}$ and GANT61-10 $\mu \mathrm{M}$, respectively, $\mathrm{n}=3$ ). Each group was analyzed in triplicate, and the data are presented as the average \pm SD.

the length and $\mathrm{W}$ the width of the tumor. A portion of each tumor was used for western blot analysis for LC3II.

Statistical analysis. All experiments were repeated in triplicate. All values are expressed as the means \pm standard deviation (SD). Statistical significance was determined by a Student's t-test using SPSS 15.0. P-values $<0.05$ were considered to indicate statistically significant differences.

\section{Results}

Effects of N-Shh and GANT61 on the expression of Gli-1 and Gli-2 in CFPAC-1 cells. To determine N-Shh (a recombinant Shh ligand) and GANT61 (a small-molecule inhibitor of Gli-1 and Gli-2) function in CFPAC-1 cells, the cells were treated with N-Shh $(0.25 / 0.5 \mu \mathrm{g} / \mathrm{ml})$ or GANT61 $(5 / 10 \mu \mathrm{M})$ for 12,24 and $48 \mathrm{~h}$, respectively. The expression levels of Gli-1 and Gli-2 were confirmed by qRT-PCR. Our data showed that N-Shh increased the mRNA levels of Gli-1 and Gli-2 while GANT61 decreased the Gli-1 and Gli-2 mRNA levels (Fig. 1). Gli-1 and Gli-2 mRNA levels were markedly upregulated by $0.5 \mu \mathrm{g} / \mathrm{ml}$ $\mathrm{N}-$ Shh at $48 \mathrm{~h}$ and were markedly downregulated by $10 \mu \mathrm{M}$ GANT61 at $48 \mathrm{~h}$, respectively. Therefore, N-Shh was used at a concentration of $0.5 \mu \mathrm{g} / \mathrm{ml}$ while GANT61 was used at $10 \mu \mathrm{M}$ in the subsequent experiments.

HH signaling regulates autophagy in CFPAC-1 cells. LC3 is widely used to monitor autophagy. The density of the LC3-II band divided by the density of the tubulin band represents the expression level of LC3-II. The ratio of the LC3-II level/ tubulin level was used as an indicator of the autophagic level. In our experiment, CFPAC-1 cells were transfected with $\mathrm{N}-\mathrm{Shh}$ or GANT61 for $48 \mathrm{~h}$. The cells were then treated with bafilomycin (400 nM; Sigma), which significantly inhibits autophagy, for $2 \mathrm{~h}$ and used as an autophagy inhibitor.

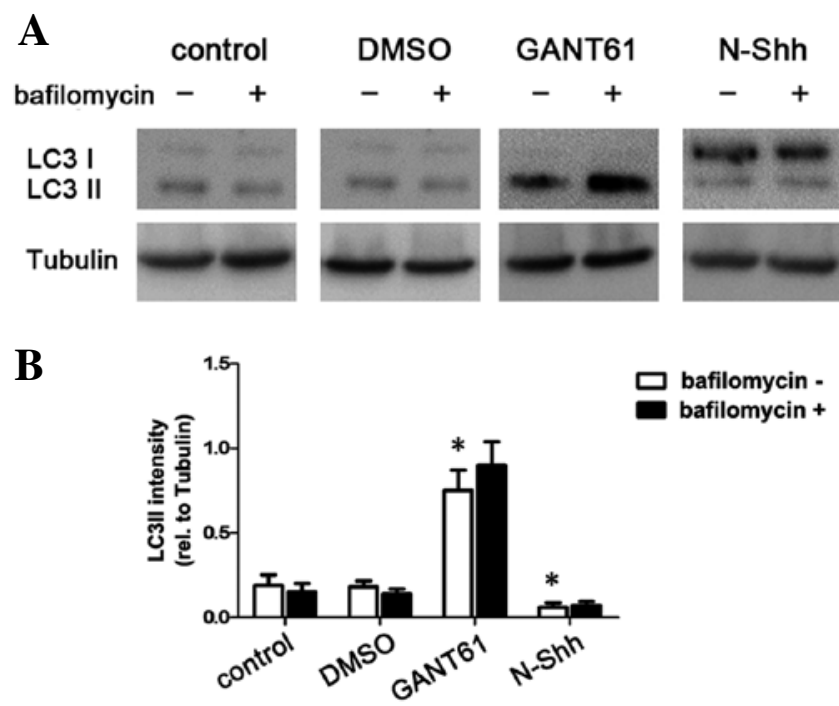

Figure 2. HH signaling regulates autophagy. (A) Western blotting for LC3 using lysates from CFPAC-1 cells treated with N-Shh or GANT61. Nontreated cells and vehicle group (DMSO) were used as controls. The ratio of the LC3-II level/tubulin level was used as an indicator of the autophagic level. (B) The ratio of LC3-II/tubulin is presented in the bar chart $\left({ }^{*} \mathrm{P}<0.05\right.$ compared with the control group, $\mathrm{n}=3$ ).

Non-treated cells and the vehicle group (DMSO) were used as controls. Western blot analysis showed that, compared with the non-treated cells, N-Shh reduced the ratio of the LC3-II level/tubulin level $(\mathrm{P}<0.05$; Fig. $2 \mathrm{~A}$ and $\mathrm{B})$, indicating that the process of autophagy was inhibited. Meanwhile, GANT61 significantly elevated the ratio of the LC3-II level/tubulin level $(\mathrm{P}<0.05$; Fig. 2A and $\mathrm{B})$. These data suggest that activation of $\mathrm{HH}$ signaling reduces autophagy, while inhibition of $\mathrm{HH}$ signaling induces autophagy. Therefore, $\mathrm{HH}$ signaling regulates autophagy in pancreatic cancer CFPAC-1 cells. 

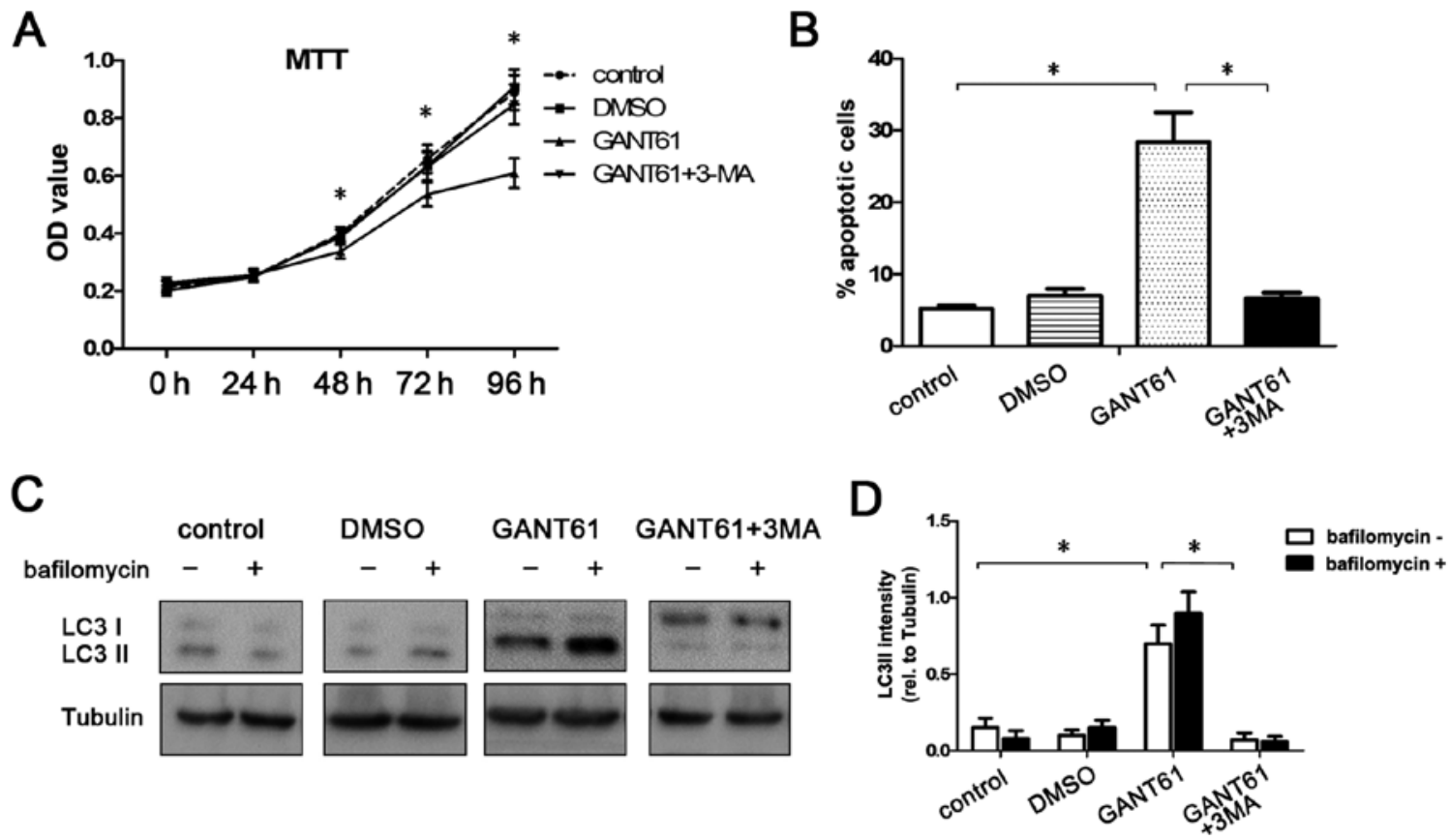

Figure 3. GANT61-induced autophagy contributes to reduced viability and increased apoptosis in pancreatic cancer cells. (A) The viability of CFPAC-1 cells treated with GANT61, GANT61 + 3-MA or vehicle over $96 \mathrm{~h}$ was measured using MTT assays ("P<0.05; $\mathrm{n}=3$ ). (B) Cell apoptosis was assessed by flow cytometry. GANT61 treated cells showed a higher apoptosis ratio $(28.38 \pm 4.12 \%)$, whereas the GANT61 + 3-MA group exhibited a lower apoptosis ratio $\left(6.59 \pm 0.81 \%,{ }^{*} \mathrm{P}<0.05 ; \mathrm{n}=3\right)$. (C) Western blotting for LC3 using lysates from CFPAC-1 cells treated with GANT61 or GANT61 + 3-MA. Non-treated cells and vehicle group (DMSO) were used as controls. The ratio of the LC3-II level/tubulin level was used as an indicator of the autophagic level. (D) The ratio of LC3-II/tubulin is presented in the bar chart $(* \mathrm{P}<0.05, \mathrm{n}=3)$.

GANT61-induced autophagy contributes to induction of apoptosis. In pancreatic cancer, it remains unclear whether autophagy acts fundamentally as a cell survival or cell death pathway. Cell viability and apoptosis were analyzed after treatment with $10 \mu \mathrm{M}$ GANT61 for $48 \mathrm{~h}$ to investigate whether GANT61-induced autophagy contributes to cell survival or death. MTT and flow cytometric assay showed that GANT61 decreased the viability and enhanced the apoptosis of CFPAC-1 cells when compared with the control group $(\mathrm{P}<0.05$; Fig. $3 \mathrm{~A}$ and B).

Since both HH signaling and autophagy involve complex crosstalks with many other pathways, we used 3-MA (an inhibitor of autophagy) to inhibit autophagy to confirm whether or not GANT61-induced apoptosis is caused by the activation of autophagy. Our data showed that GANT61-induced apoptosis and reduction in cell viability in CFPAC- 1 cells were reversed by inhibition of autophagy by $3-\mathrm{MA}(\mathrm{P}<0.05$; Fig. 3A and B). Western blot analysis showed that, compared with the GANT61-treated group, the ratio of the LC3-II level/tubulin level was significantly reduced in the group treated with the combination of GANT61 and 3-MA (P<0.05; Fig. 3C and D), suggesting the GANT61-induced autophagy was inhibited by 3-MA. These results suggest that GANT61-induced autophagy contributes to the viability and apoptosis of CFPAC-1 cell.

GANT61 induces autophagy and suppresses pancreatic cancer cell growth in vivo. To further investigate the antitumor potential of GANT61 and the role of autophagy in vivo, $\mathrm{BALB} / \mathrm{c}$ nude mice were injected undercapsule of the pancreas with CFPAC-1 cells. Vehicle (DMSO), GANT61 $(50 \mathrm{mg} / \mathrm{kg})$, GANT61 $(50 \mathrm{mg} / \mathrm{kg})$ in combination with 3-MA $(10 \mathrm{mg} / \mathrm{kg})$ were administered every other day, i.p. for 28 days (starting one week after inoculation). The data revealed that GANT61 significantly inhibited tumor growth in CFPAC-1 cells when compared with the control group $(\mathrm{P}<0.05$; Fig. $4 \mathrm{~A}$ and $\mathrm{B})$ and the effect was reversed by co-treatment with 3-MA, the autophagy inhibitor. Since the formation of special doublemembraned structures containing undigested cytoplasmic contents (autophagosomes) is the most important characteristic of autophagy, detecting these structures by electron microscopy is considered the gold standard for documenting autophagy. Hence, we examined the ultrastructural changes in the orthotopic xenograft tumors. Quantification of autophagosomes revealed that the number of autophagosomes was significantly increased in the GANT61 treatment group $(9.26 \pm 2.54)$; however, the number of autophagosomes was reduced in the combination group $(5.13 \pm 1.97)(\mathrm{P}<0.05$; Fig. $4 \mathrm{C}$ and $\mathrm{D})$. These results indicate that GANT61-induced autophagy contributes to the viability of CFPAC-1 cells in vivo.

\section{Discussion}

In the present study, we demonstrated that the HH signaling pathway regulates autophagy in pancreatic cancer cells. Our data showed that activation of HH signaling by its ligand, $\mathrm{N}$-Shh, inhibits autophagy, while inhibition of the HH pathway by GANT61 induces autophagy. In addition, GANT61-induced autophagy contributed to reduced viability and increased apoptosis in CFPAC-1 cells and these effects were reversed by the co-treatment with 3-MA, the autophagy inhibitor.

The HH signaling pathway is a 'core' signal transduction pathway in pancreatic cancer (10) that promotes the 


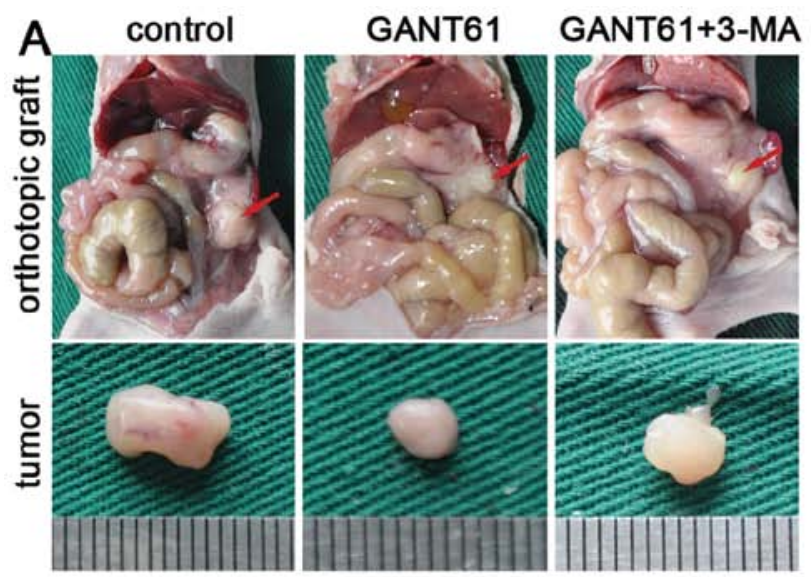

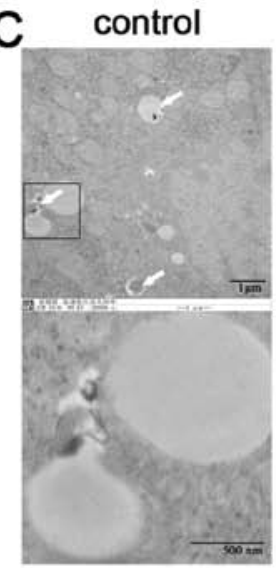

GANT61

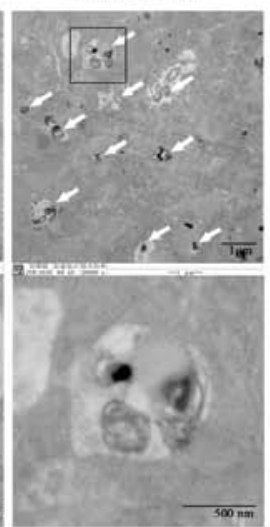

GANT61+3-MA

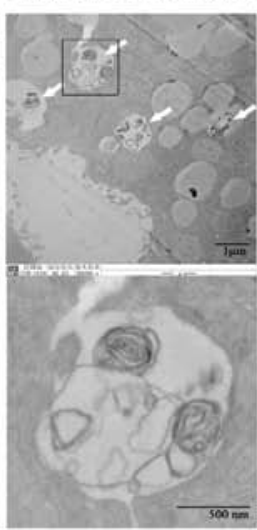

D

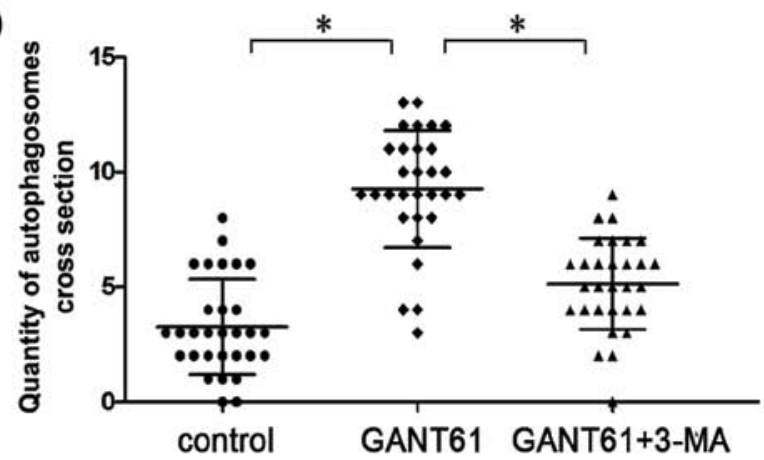

Figure 4. GANT61 induces autophagy and suppresses pancreatic cancer cell growth in vivo. (A) Orthotopic xenograft model of pancreatic cancer and gross specimens of tumors after receiving treatment with GANT61 or GANT61 + 3-MA. The images are representative of 6 specimens accordingly. (B) Tumor volume of the three groups $\left({ }^{*} \mathrm{P}<0.05 ; \mathrm{n}=6\right)$. GANT61 treatment significantly inhibited CFPAC-1 tumor growth, and the effectiveness was attenuated by the autophagy inhibitor, 3-MA. (C) Induction of autophagy in GANT61-treated tumors was confirmed by transmission electron microscopy. The white arrows indicate the double membrane-surrounded autophagosomes in the tumor samples. Upper panel, overview images (magnification, $\mathrm{x} 20,000$ ). Scale bars represent $1 \mu \mathrm{m}$. Lower panel, enlarged images of cytoplasmic regions of the upper panel indicated within the dashed lines. Scale bars represent $500 \mathrm{~nm}$. (D) The data were quantified by counting the number of autophagosomes per cross-sectioned cell $\left({ }^{*} \mathrm{P}<0.05 ; \mathrm{n}=30\right)$.

tumorigenesis of pancreatic cancers via enhancing cell proliferation $(11,12)$, increasing invasion and metastasis $(13,14)$ and protecting against apoptosis $(15,16)$. Therapies targeting $\mathrm{HH}$ signaling were believed to be an effective method through which to overcome pancreatic cancer. However, in a phase I trial testing vismodegib (GDC-0449), an inhibitor of Smoothened (SMO), no beneficial responses were observed in pancreatic carcinoma (17). The ineffectiveness of the pathway inhibitor for pancreatic cancer was probably due to the fact there is much crosstalk between the $\mathrm{HH}$ signaling pathway and other signaling pathways in pancreatic cancer (18-20). In the present study, we found that $\mathrm{HH}$ signaling regulated autophagy in pancreatic cancer cells. Our data revealed that activation of $\mathrm{HH}$ signaling by its ligand, N-Shh, inhibited autophagy, while inhibition of the HH pathway by GANT61 induced autophagy.

The role of autophagy in pancreatic cancer development and progression is complex. During the development of pancreatic cancer, autophagy initially suppresses tumor initiation yet supports tumor growth at later stages $(27,28)$. Many results suggest that autophagy is a cell death mechanism in response to drugs such as gemcitabine and triptolide. However, it was also reported that activation of autophagy promotes tumorigenesis and limits apoptosis in pancreatic cancer cells. In the present study, we found that GANT61induced autophagy contributed to reduced viability and increased apoptosis in CFPAC-1 cells both in vivo and in vitro, and these effects were reversed by the blockage of autophagy. Our findings were confirmed by a recent study in which Wang et al (39) found that the $\mathrm{HH}$ signaling pathway regulates autophagy in HCC, inhibition of Gli by GANT61 induces autophagy, and blockage of autophagy attenuates GANT61-induced apoptosis. However, in a recent study, we found that miR-101 enhanced apoptosis induced by cisplatin in HCC cells by inhibition of autophagy (40). Although these findings appear contradictory, these inconsistent conclusions confirm that autophagy plays a dual role in tumor initiation and development. The tumor-suppressor or tumor-promoter role depends on the type of cancer and the microenvironment. In addition, inhibition of autophagy by different approaches caused inconsistent effects which may be related to the complex crosstalk between autophagy and many other pathways. Different methods of autophagy inhibition may regulate different crosstalk pathways resulting in a different degree of effectiveness. Thus, further investigation of the mechanism of interaction between autophagy and these crosstalk pathways is particularly important.

In conclusion, the present study showed that $\mathrm{HH}$ signaling regulates autophagy in pancreatic cancer cells. Activation of $\mathrm{HH}$ signaling inhibits autophagy, while inhibition of the $\mathrm{HH}$ pathway induces autophagy. Although the role of autophagy 
in cell survival and apoptosis may depend on tumor type and microenvironment, our data clearly demonstrated that GANT61-induced autophagy contributes to reduced viability and increased apoptosis in pancreatic cancer cells both in vivo and in vitro. We propose that $\mathrm{HH}$ signaling by regulating autophagy plays an important role in determining the cellular response to $\mathrm{HH}$-targeted therapy in pancreatic cancer and further investigation concerning the interaction between autophagy and $\mathrm{HH}$ signaling is particularly important.

\section{Acknowledgements}

The present study was supported by grants from the Department of Public Health of Jiangsu Province (no. RC2007056) and the National Natural Science Foundation of China (no. 81170415).

\section{References}

1. Siegel R, Naishadham D and Jemal A: Cancer statistics, 2013. CA Cancer J Clin 63: 11-30, 2013.

2. Hammerschmidt M, Brook A and McMahon AP: The world according to hedgehog. Trends Genet 13: 14-21, 1997.

3. Varjosalo $M$ and Taipale J: Hedgehog: functions and mechanisms. Genes Dev 22: 2454-2472, 2008.

4. Kalderon D: Transducing the hedgehog signal. Cell 103: 371-374, 2000.

5. McMahon AP: More surprises in the Hedgehog signaling pathway. Cell 100: 185-188, 2000.

6. Caro I and Low JA: The role of the hedgehog signaling pathway in the development of basal cell carcinoma and opportunities for treatment. Clin Cancer Res 16: 3335-3339, 2010.

7. Barginear MF, Leung $M$ and Budman DR: The hedgehog pathway as a therapeutic target for treatment of breast cancer. Breast Cancer Res Treat 116: 239-246, 2009.

8. Saqui-Salces $M$ and Merchant JL: Hedgehog signaling and gastrointestinal cancer. Biochim Biophys Acta 1803: 786-795, 2010.

9. Thayer SP, di Magliano MP, Heiser PW, et al: Hedgehog is an early and late mediator of pancreatic cancer tumorigenesis Nature 425: 851-856, 2003.

10. Jones S, Zhang X, Parsons DW, et al: Core signaling pathways in human pancreatic cancers revealed by global genomic analyses. Science 321: 1801-1806, 2008.

11. Yauch RL, Dijkgraaf GJ, Alicke B, et al: Smoothened mutation confers resistance to a Hedgehog pathway inhibitor in medulloblastoma. Science 326: 572-574, 2009.

12. Morton JP, Mongeau ME, Klimstra DS, et al: Sonic hedgehog acts at multiple stages during pancreatic tumorigenesis. Proc Natl Acad Sci USA 104: 5103-5108, 2007.

13. Bailey JM, Mohr AM and Hollingsworth MA: Sonic hedgehog paracrine signaling regulates metastasis and lymphangiogenesis in pancreatic cancer. Oncogene 28: 3513-3525, 2009.

14. Dai J, Ai K, Du Y and Chen G: Sonic hedgehog expression correlates with distant metastasis in pancreatic adenocarcinoma. Pancreas 40: 233-236, 2011.

15. Olive KP, Jacobetz MA, Davidson CJ, et al: Inhibition of Hedgehog signaling enhances delivery of chemotherapy in a mouse model of pancreatic cancer. Science 324: 1457-1461, 2009.

16. Lonardo E, Hermann PC, Mueller MT, et al: Nodal/activin signaling drives self-renewal and tumorigenicity of pancreatic cancer stem cells and provides a target for combined drug therapy. Cell Stem Cell 9: 433-446, 2011.

17. LoRusso PM, Rudin CM, Reddy JC, et al: Phase I trial of hedgehog pathway inhibitor vismodegib (GDC-0449) in patients with refractory, locally advanced or metastatic solid tumors. Clin Cancer Res 17: 2502-2511, 2011.
18. Pasca di Magliano M, Sekine S, Ermilov A, Ferris J, Dlugosz AA and Hebrok M: Hedgehog/Ras interactions regulate early stages of pancreatic cancer. Genes Dev 20: 3161-3173, 2006.

19. Kasperczyk H, Baumann B, Debatin KM and Fulda S: Characterization of sonic hedgehog as a novel NF- $\kappa \mathrm{B}$ target gene that promotes NF- $\mathrm{BB}$-mediated apoptosis resistance and tumor growth in vivo. FASEB J 23: 21-33, 2009.

20. Eberl M, Klingler S, Mangelberger D, et al: Hedgehog-EGFR cooperation response genes determine the oncogenic phenotype of basal cell carcinoma and tumour-initiating pancreatic cancer cells. EMBO Mol Med 4: 218-233, 2012.

21. Ashford TP and Porter KR: Cytoplasmic components in hepatic cell lysosomes. J Cell Biol 12: 198-202, 1962.

22. Mizushima N, Levine B, Cuervo AM and Klionsky DJ: Autophagy fights disease through cellular self-digestion. Nature 451: 1069-1075, 2008.

23. Levine B and Kroemer G: Autophagy in the pathogenesis of disease. Cell 132: 27-42, 2008.

24. Lorin S, Hamai A, Mehrpour M and Codogno P: Autophagy regulation and its role in cancer. Semin Cancer Biol 23: 361-379, 2013.

25. White E: Deconvoluting the context-dependent role for autophagy in cancer. Nat Rev Cancer 12: 401-410, 2012.

26. Eskelinen EL: The dual role of autophagy in cancer. Curr Opin Pharmacol 11: 294-300, 2011.

27. Yang S and Kimmelman AC: A critical role for autophagy in pancreatic cancer. Autophagy 7: 912-913, 2011.

28. Aghajan M, Li N and Karin M: Obesity, autophagy and the pathogenesis of liver and pancreatic cancers. J Gastroenterol Hepatol 27 (Suppl 2): 10-14, 2012.

29. Yang S, Wang X, Contino G, et al: Pancreatic cancers require autophagy for tumor growth. Genes Dev 25: 717-729, 2011.

30. Fujii S, Mitsunaga S, Yamazaki M, et al: Autophagy is activated in pancreatic cancer cells and correlates with poor patient outcome. Cancer Sci 99: 1813-1819, 2008.

31. Mukubou H, Tsujimura T, Sasaki R and Ku Y: The role of autophagy in the treatment of pancreatic cancer with gemcitabine and ionizing radiation. Int J Oncol 37: 821-828, 2010.

32. Donadelli M, Dando I, Zaniboni T, et al: Gemcitabine/cannabinoid combination triggers autophagy in pancreatic cancer cells through a ROS-mediated mechanism. Cell Death Dis 2: e152, 2011.

33. Kang R, Tang D, Schapiro NE, et al: The receptor for advanced glycation end products (RAGE) sustains autophagy and limits apoptosis, promoting pancreatic tumor cell survival. Cell Death Differ 17: 666-676, 2010.

34. Kang R, Tang D, Lotze MT and Zeh HJ III: AGER/RAGEmediated autophagy promotes pancreatic tumorigenesis and bioenergetics through the IL6-pSTAT3 pathway. Autophagy 8: 989-991, 2012.

35. Xi H, Kurtoglu M, Liu H, et al: 2-Deoxy-D-glucose activates autophagy via endoplasmic reticulum stress rather than ATP depletion. Cancer Chemother Pharmacol 67: 899-910, 2011.

36. Mirzoeva OK, Hann B, Hom YK, et al: Autophagy suppression promotes apoptotic cell death in response to inhibition of the PI3K-mTOR pathway in pancreatic adenocarcinoma. J Mol Med (Berl) 89: 877-889, 2011.

37. Wu CY, Yan J, Yang YF, et al: Overexpression of KAI1 induces autophagy and increases $\mathrm{MiaPaCa}-2$ cell survival through the phosphorylation of extracellular signal-regulated kinases. Biochem Biophys Res Commun 404: 802-808, 2011.

38. Livak KJ and Schmittgen TD: Analysis of relative gene expression data using real-time quantitative PCR and the 2(-Delta Delta C(T)) Method. Methods 25: 402-408, 2001.

39. Wang Y, Han C, Lu L, Magliato S and Wu T: Hedgehog signaling pathway regulates autophagy in human hepatocellular carcinoma cells. Hepatology 58: 995-1010, 2013.

40. Xu Y, An Y, Wang Y, et al: miR-101 inhibits autophagy and enhances cisplatin-induced apoptosis in hepatocellular carcinoma cells. Oncol Rep 29: 2019-2024, 2013. 